\title{
Phase transitions in hexane monolayers physisorbed onto graphite
}

\author{
M. W. Roth and C. L. Pint \\ Department of Physics, University of Northern Iowa, Cedar Falls, Iowa 50614, USA \\ Carlos Wexler \\ Department of Physics and Astronomy, University of Missouri-Columbia, Columbia, Missouri 65211, USA
}

(Received 15 November 2004; published 29 April 2005)

\begin{abstract}
We report the results of molecular dynamics simulations of a complete monolayer of hexane physisorbed onto the basal plane of graphite. At low temperatures the system forms a herringbone solid. With increasing temperature, a solid-to-nematic liquid-crystal transition takes place at $T_{1}=138 \pm 2 \mathrm{~K}$ followed by another transition at $T_{2}=176 \pm 3 \mathrm{~K}$ into an isotropic fluid. We characterize the different phases by calculating various order parameters, coordinate distributions, energetics, spreading pressure, and correlation functions, most of which are in reasonable agreement with available experimental evidence. In addition, we perform simulations where the Lennard-Jones interaction strength, corrugation potential strength, and dihedral rigidity are varied in order to better characterize the nature of the two transitions. We find that both phase transitions are facilitated by a "footprint reduction" of the molecules via tilting and to a lesser degree via creation of gauche defects in the molecules.
\end{abstract}

DOI: 10.1103/PhysRevB.71.155427

PACS number(s): 64.70. $-\mathrm{p}, 68.35 . \mathrm{Rh}, 68.43 .-\mathrm{h}$

\section{INTRODUCTION}

The physical adsorption (physisorption) of atoms and molecules onto a substrate to form a quasi-two-dimensional (2D) film has resulted in the observation of a rich variety of behavior not realized in the system's corresponding threedimensional (3D) or "bulk" state.

Among the species used as adsorbates, alkanes are of considerable interest because of their technological importance (e.g., as lubricants), and because they are among the simplest families of molecules of compounds whose members differ mainly in their lengths. In fact, straight-chained $n$ alkanes represent a fine balance between complexity (i.e., many internal degrees of freedom, which also affect the interaction between molecules) and the simplicity of their structure (as compared to other organic molecules). In fact, over the past decades there has been a constant and renewed effort to better understand the adsorption of alkanes over a variety of substrates. Among the substrates used for physisorption studies, graphite, with its excellent mechanical stability, availability, and symmetry, has proven to be one of the best choices. A considerable amount of experimental, theoretical, and computational work has, thus, been devoted to these studies.

Hexane on graphite was first studied by Krim et al. ${ }^{1}$ using low-energy electron diffraction (LEED) as well as neutron diffraction. For submonolayer coverage a uniaxialincommensurate (UI) herringbone phase is observed below approximately $T=151 \mathrm{~K}$, where a first-order melting transition is found (but note that a determination of the molecular orientations was not possible). At low temperatures, as the coverage is increased, the UI phase evolves continuously into a $2 \times 4 \sqrt{3}$ commensurate structure at completion. More recently, Taub ${ }^{3}$ completed extensive neutron ${ }^{2,3}$ and $\mathrm{x}$-ray ${ }^{2}$ diffraction studies of hexane on graphite for submonolayer, ${ }^{2}$ monolayer, ${ }^{2-6}$ and multilayer ${ }^{2}$ coverages. Their findings indicate that at low temperatures a complete monolayer forms a commensurate herringbone structure which evolves with increasing temperature into a rectangular centered solid/liquid coexistence region by approximately $150 \mathrm{~K}$ and melts at around $175 \mathrm{~K}^{2-6}$ For submonolayer coverages, these authors proposed a structure corresponding to a UI phase comprised of commensurate regions separated by low-density fluid filled domain walls. ${ }^{2}$

In the 1990s, Hansen and co-workers reported studies combining molecular dynamics (MD) computer simulations with neutron and $\mathrm{x}$-ray diffraction studies of butane and hexane on graphite at monolayer completion. ${ }^{4,5}$ Experimentally, butane melts abruptly at around $T=116 \mathrm{~K}$, directly from a solid, commensurate, rectangular-centered herringbone (HB) phase into a liquid. Orientational ordering about the surface normal is lost through rotation about the center of mass, which is simultaneous with melting. Hexane, on the other hand, undergoes a loss of translational order at about $150 \mathrm{~K}$ into a phase with short-range order that is thought to involve mobile rectangular-centered (RC) islands within a fluid. Then, at around $T=175 \mathrm{~K}$ the system melts into a fluid.

The computer simulations of Hansen and co-workers ${ }^{4,5}$ showed that, for hexane, the internal formation of gauche defects coupled with out-of-plane tilting is concurrent with melting. The necessary creation of in-plane room by either gauche defects or tilting at the onset of melting is referred to as a "footprint reduction" mechanism. These simulations showed, furthermore, that the nature and temperature of the transition is very sensitive to the presence of gauche defects, as the melting temperature rises drastically when gauchedefect formation is suppressed. However, the resulting melting temperature of a hexane monolayer was found to be $222 \mathrm{~K}$, significantly different from the experimental value of $175 \mathrm{~K}$. This discrepancy was addressed in a series of MD simulations of hexane on graphite by Velasco and Peters ${ }^{7}$ who found a better agreement with experimental values of the melting temperature when a considerably lower adsorbate-interaction strength was used, and under those 
conditions molecular gauche-defect formation becomes almost irrelevant to melting. (In this paper we find the presence of an intermediate nematic phase. In the herringbone solid-to-nematic phase transition we find gauche defects to be irrelevant. For the nematic-to-isotropic-liquid phase transition, we find that both mechanisms contribute to the footprint reduction, although it is apparent that tilting is the dominant effect.)

More recently, Peters and co-workers have also examined the behavior of monolayer ${ }^{8}$ and bilayer ${ }^{9}$ hexane on graphite using MD simulation techniques. The monolayer study compared MD results based on the standard (isotropic) unified atom (UA) approximation to simulations where an anisotropic force field was employed. Using a computational cell of 112 hexane molecules and performing MD simulations corresponding to 200000 steps of $1 \mathrm{fs}$, both models yielded the same basic physics, especially in regards to the characterization of the phases observed. (The transition temperature from commensurate herringbone to the rectangular-centered orientationally ordered phase is found at around $150 \mathrm{~K}$ and the melting temperature at $175 \mathrm{~K}$ in both cases. The main difference between the two models is that the anisotropic model promotes more in-plane mobility from vacancy creation due to increased molecule tilting at all temperatures.)

Even though hexane on graphite has been studied quite well experimentally, it has not been as thoroughly investigated computationally, and there is still some doubt about the roles played in the phase transitions by the internal degrees of freedom of the molecules (mainly gauche defects) and their relative importance as the adatom-substrate interaction is varied. Furthermore, it is not self-evident that the simulations have achieved ergodicity to a satisfactory degree, as only a relatively limited simulation time has been explored so far (maximum of $2 \mathrm{~ns}$ in Ref. 8). The general purpose of the work reported here is to enrich our understanding of phase transitions in physisorbed molecular systems and, in particular, of hexane in graphite. Specifically, we wish to: (i) more sharply delineate the phase transitions of hexane on graphite using longer simulations (up to 5 times longer than previously reported); (ii) understand the effects of varying the graphite corrugation strength and the adatom-adatom interaction strength on the observed phase transitions; (iii) further delineate the role of the internal degrees of freedom of the adsorbed molecules, i.e., the bond bending and dihedral torsion in the observed phase transitions; and (iv) to gain insight into the energetics and possible mechanisms for the observed phase transitions.

Since the system under consideration is, to some degree, (quasi-) two-dimensional, one may have expected that the observed phase transitions would show some of the universal features expected from the beautiful theory of 2D melting developed in the 1970s by Kosterlitz, Thouless, Halperin, Nelson and Young. ${ }^{10-14}$ However, the importance of the outof-plane behavior of the hexane monolayer and the "polarizing" effect of the substrate corrugation make it difficult to reliably place these transitions in a general theoretical context. Furthermore, it is evident that the relatively small system sizes achievable in MD simulations do not permit a complete characterization of the phase transitions observed (e.g., their order), but sometimes the modality of the energy distributions near the critical regions may give indications of what behavior to expect.

The paper is organized as follows. In Sec. II we discuss the computer simulation procedures. The results of the simulations are presented in Sec. III and are discussed in detail in Sec. IV.

\section{COMPUTATIONAL DETAILS}

The hexane molecules in our study are modeled with two methyl $\left(\mathrm{CH}_{3}\right)$ pseudoatoms on their ends and four methylene $\left(\mathrm{CH}_{2}\right)$ pseudoatoms in between. The lowest energy conformation of the molecule consists of all pseudoatoms being co-planar, forming a zizzag pattern with $114^{\circ}$ three-body bond angles (dihedral angles) and bond lengths (nearestneighbor separations) of $1.54 \AA$. In the simulations that follow we keep the bond lengths fixed, but allow the other internal degrees of freedom to vary (bond angle bending and dihedral angle torsion). The graphite substrate modeled as being static, contains an infinite number of stacked graphene sheets (see Fig. 1). The structure contains point carbon atoms at the vertices of all bonds and the bond length is taken to be $2.46 \AA$.

\section{A. Method}

A constant particle number, planar density, and temperature $(N=672, \rho=1, T)$ molecular dynamics (MD) method is employed to conduct atomistic simulations in a system of $N_{m}=2 \times m \times n=112$ hexane molecules at monolayer completion $(m=4$ and $n=14)$. The rectangular computational cell (see Fig. 1) has its corners coincident with graphite hexagon centers and has dimensions $m \times 4 \sqrt{3} a \simeq 68.1735 \AA$ and $n$ $\times 2 a=68.88 \AA$ in the $x$ and $y$ directions, respectively. Such a cell gives a planar density of $\rho=0.02385$ molecules $/ \AA^{2}$ and an area per molecule of $42 \AA^{2}$. Each hexane molecule consists of six united atom (UA) pseudoatoms - two methyl $\left(\mathrm{CH}_{3}\right)$ and four methylene $\left(\mathrm{CH}_{2}\right)$. Periodic boundary conditions are utilized for each pseudoatom in the $x, y$ directions and free boundary conditions are present in the vertical $z$ direction. To perform the simulations at constant temperature, we periodically rescale the velocities so that equipartition is satisfied for the center-of-mass, rotational, and internal temperatures,

$$
\begin{gathered}
T_{C M}=\frac{1}{3 N_{m} k_{B}} \sum_{i=1}^{N_{m}} M_{i} v_{i, C M}^{2}, \\
T_{R O T}=\frac{1}{3 N_{m} k_{B}} \sum_{i=1}^{N_{m}} \boldsymbol{\omega}_{i}^{T} \mathcal{I}_{i} \boldsymbol{\omega}_{i}, \\
T_{I N T}=\frac{1}{\left(2 n_{C}-5\right) N_{m} k_{B}} \sum_{i=1}^{N_{m}} \sum_{j=1}^{n_{C}} m_{i j}\left[\left(\mathbf{v}_{i j}-\mathbf{v}_{i, C M}\right)-\boldsymbol{\omega}_{i}\right. \\
\left.\times\left(\mathbf{r}_{i j}-\mathbf{r}_{i, C M}\right)\right]^{2},
\end{gathered}
$$

respectively. (No significant differences were found when "thermalizing" a subset of the temperatures above.) Here $N_{m}$ 

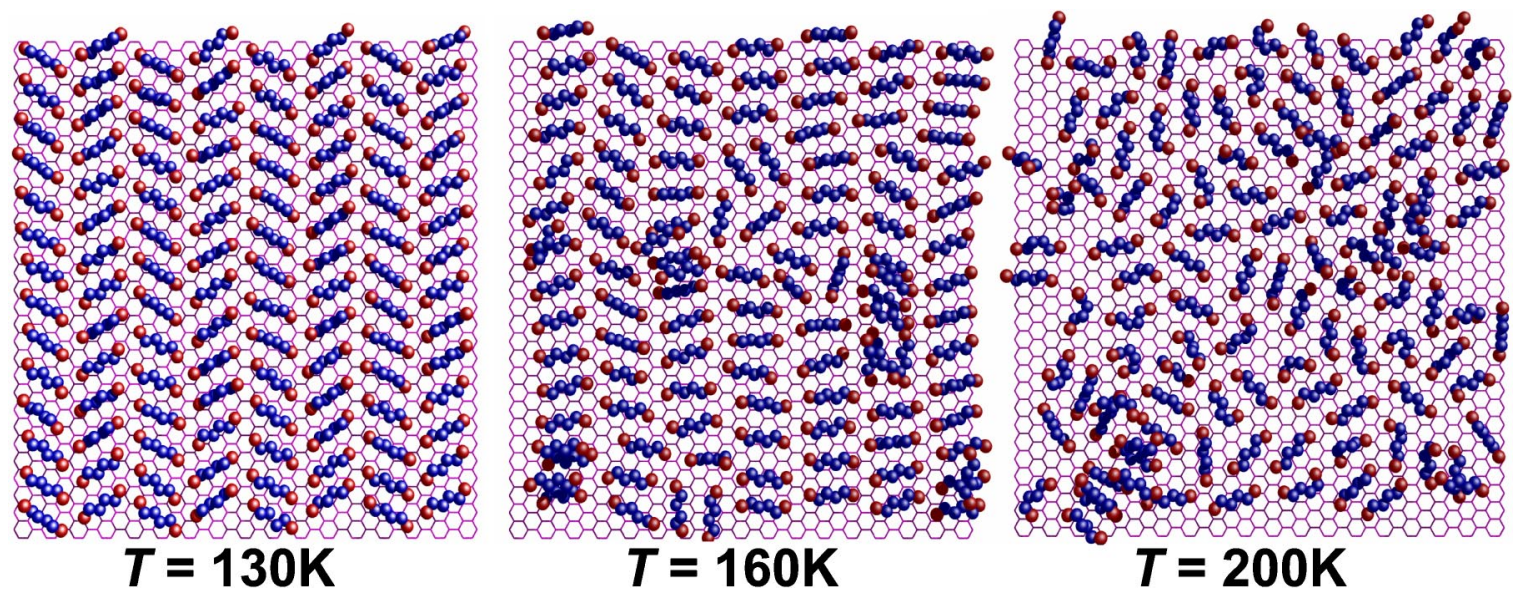

FIG. 1. (Color online) Simulation cell showing snapshots of typical (equilibrium) configurations for the hexane/graphite system in the commensurate herringbone solid $(T=130 \mathrm{~K})$, the orientationally ordered nematic $(T=160 \mathrm{~K})$, and the isotropic liquid $(T=200 \mathrm{~K})$ phases. $\mathrm{CH}_{3}$ end-of-chain pseudoatoms are red, $\mathrm{CH}_{2}$ are yellow, and the $\mathrm{C}-\mathrm{C}$ bonds in the graphite substrate are purple (the graphite lattice spacing is $a=2.46 \AA$ ). The computational cell has dimensions $m \times 4 \sqrt{3} a \simeq 68.1735 \AA$ and $n \times 2 a=68.88 \AA$ in the $x$ and $y$ directions respectively, where $m=4$ and $n=14$, and periodic boundary conditions are used in $x$ and $y$ directions. There are $2 \times m \times n=112$ hexane molecules.

is the number of molecules, and $n_{C}$ is the number of pseudoatoms per molecule; the $i$ index runs over the molecules $\left(1 \leqslant i \leqslant N_{m}\right)$, and the $j$ index runs over the pseudoatoms within a given molecule $\left(1 \leqslant j \leqslant n_{C}\right)$. Furthermore, for each molecule, $M_{i}$ is the mass, $\mathbf{v}_{i, C M}$ is the center-of-mass velocity, $\boldsymbol{\omega}_{i}$ is the angular velocity, and $\mathcal{I}_{i}$ is the moment of inertia tensor [relative to the center of mass (COM)]. Last, $\mathbf{v}_{i j}$ is the velocity of the $j$ th pseudoatom in the $i$ th molecule, and $k_{B}$ is Boltzmann's constant.

The time step for all simulations is chosen to be $1 \mathrm{fs}$, and integration of the equations of motion is achieved with a velocity Verlet RATTLE $^{15}$ ) algorithm which keeps the pseudoatom bond lengths constant. The runs are typically started from a herringbone configuration, as suggested by experimental evidence (e.g., the neutron diffraction data of Ref. 1), and allowed to equilibrate for roughly $3-5 \times 10^{5}$ steps; averages are taken for $0.5-1.0 \times 10^{6}$ steps after equilibration. Some runs are started from the final configuration of some lower temperature runs. A good measure of convergence is that incorporating the results from both types of initial conditions does not create noticeable scatter in the results. The only hysteresis observed is that, as is usual in these type of simulations, "freezing" by starting from a hightemperature phase and lowering the temperature is not readily achieved.

\section{B. Interaction potentials}

The model adopted for our study of adsorbed hexane molecules on graphite corresponds to having both nonbonded and bonded interactions. The first of two nonbonded interactions is the adsorbate-adsorbate interaction between pseudoatoms $i$ and $j$ separated by a distance $r_{i j}$, which is modeled by a Lennard-Jones pair potential,

$$
u_{L J}\left(r_{i j}\right)=4 \epsilon_{i j}\left[\left(\frac{\sigma_{i j}}{r_{i j}}\right)^{12}-\left(\frac{\sigma_{i j}}{r_{i j}}\right)^{6}\right] .
$$

Lorentz-Bertholot combining rules,

$$
\sigma_{i j}=\frac{\sigma_{i}+\sigma_{j}}{2}, \quad \epsilon_{i j}=\sqrt{\epsilon_{i} \epsilon_{j}}
$$

are applied in order to describe mixed interactions when particles $i$ and $j$ are of different types; lattice sums are taken out to $10 \AA$, and values for the potential parameters used in our work ${ }^{16}$ are given in Table I.

The second nonbonded interaction is the adatom-graphite interaction. The graphite is modeled as being of infinite extent in the $x y$ plane and semi-infinite in the vertical $-z$ direction. The adatom-graphite interaction potential, however, is not taken as a discrete sum over the carbon atoms. Steele's expansion ${ }^{17}$ is utilized because it exploits the fact that the symmetry of the substrate breaks the adatom-graphite interaction into a vertically (laterally averaged) portion and a much weaker portion related to the undulation in the interaction due to the graphite hexagonal symmetry. Such a representation saves a considerable amount of computing time. In other words, the interaction of the adsorbate atoms with the substrate is the Fourier expansion,

TABLE I. Nonbonded potential parameters used in the simulations.

\begin{tabular}{cc}
\hline \hline Parameter & Value \\
\hline$\epsilon_{\mathrm{CH}_{2}}, \epsilon_{\mathrm{CH}_{3}}$ & $72 \mathrm{~K}$ \\
$\sigma_{\mathrm{CH}_{2}}, \sigma_{\mathrm{CH}_{3}}$ & $3.92 \AA$ \\
$q$ & 2.0 \\
$a$ & $2.46 \AA$ \\
$a_{s}$ & $5.24 \AA$ \\
$d$ & $3.357 \AA$ \\
$\epsilon_{g r}$ & $44.89 \mathrm{~K}$ \\
$\sigma_{g r}$ & $3.66 \AA$ \\
\hline \hline
\end{tabular}




$$
u_{g r}\left(\mathbf{r}_{i}\right)=E_{0 i}\left(z_{i}\right)+\sum_{n=1}^{\infty} E_{n i}\left(z_{i}\right) f_{n}\left(x_{i} y_{i}\right),
$$

with

$$
\begin{aligned}
E_{0 i}\left(z_{i}\right)= & \frac{2 \pi q \epsilon_{g r} \sigma_{g r}^{6}}{a_{s}}\left[\frac{2 \sigma_{g r}^{6}}{45 d\left(z_{i}+0.72 d\right)^{9}}+\frac{2 \sigma_{g r}^{6}}{5 z_{i}^{10}}-\frac{1}{z_{i}^{4}}\right. \\
& \left.-\frac{2 z_{i}^{2}+7 z_{i} d+7 d^{2}}{6 d\left(z_{i}+d\right)^{5}}\right], \\
E_{\mathrm{ni}}\left(z_{i}\right)= & \frac{2 \pi \epsilon_{g r} \sigma_{g r}^{6}}{a_{s}}\left[\left(\frac{\sigma_{g r}^{6}}{30}\right)\left(\frac{g_{n}}{2 z_{i}}\right)^{5} K_{5}\left(g_{n} z_{i}\right)\right. \\
& \left.-2\left(\frac{g_{n}}{2 z_{i}}\right)^{2} K_{2}\left(g_{n} z_{i}\right)\right], \\
f_{1}\left(x_{i}, y_{i}\right)= & 2 \cos \left[\frac{2 \pi}{a}\left(x+\frac{y}{\sqrt{3}}\right)\right]+2 \cos \left[\frac{2 \pi}{a}\left(x-\frac{y}{\sqrt{3}}\right)\right] \\
& +2 \cos \left[\frac{4 \pi}{a}\left(\frac{y}{\sqrt{3}}\right)\right] .
\end{aligned}
$$

Here $g_{n}$ is the modulus of the $n$th graphite reciprocal-lattice vector and the $K_{n}(x)$ are modified Bessel functions of the second kind. The interaction is obtained by summing over an infinite number of graphene sheets. Only $f_{1}\left(x_{i}, y_{i}\right)$ is defined above, because the sum in the $x, y$-dependent part of Eq. (4) converges extremely rapidly and only the $n=1$ term is needed. All potential parameters for the adsorbate-substrate interaction are identified in Table I.

There are two types of bonded interactions considered in this work: bond-angle bending and dihedral-angle bending. (We assume that the bond lengths are fixed at $1.54 \AA$, and the RATTLE algorithm allows for a constrained solution of the equation of motion. ${ }^{15}$ ) The bond angles are assumed to be harmonic; their bending potential energy comes from Martin and Siepmann ${ }^{16}$ and is given by

$$
u_{\text {bend }}\left(\theta_{b}\right)=\frac{k_{\theta}}{2}\left(\theta_{b}-\theta_{0}\right)^{2}
$$

where $\theta_{b}$ is the bond angle, $\theta_{0}$ is the equilibrium bond angle, and $k_{\theta}$ is the angular stiffness. In addition, the expression for the energy of dihedral bending (torsion) $)^{8,18}$ is of the form,

$$
u_{\text {tors }}\left(\phi_{d}\right)=\sum_{i=0}^{5} c_{i}\left(\cos \phi_{d}\right)^{i},
$$

where $\phi_{d}$ is the dihedral angle and the $c_{i}$ are constants. All values for the bonded potential parameters are shown in Table II. This potential has one global minimum for the trans configuration $\phi_{d}=0$ and two local minima with $\sim 234 \mathrm{~K}$ higher in energy for the two gauche configurations at $\phi_{d}$ $= \pm 2 \pi / 3$.

The full potential energy can thus be written,

$$
U=U_{\mathrm{nb}}+U_{\mathrm{b}},
$$

TABLE II. Bonded potential parameters used in the simulations.

\begin{tabular}{cc}
\hline \hline Parameter & Value \\
\hline$k_{\theta}$ & $62793.6 \mathrm{~K} / \mathrm{rad}^{2}$ \\
$\theta_{0}$ & $114^{\circ}$ \\
$c_{0}$ & $1037.76 \mathrm{~K}$ \\
$c_{1}$ & $2426.07 \mathrm{~K}$ \\
$c_{2}$ & $81.64 \mathrm{~K}$ \\
$c_{3}$ & $-3129.46 \mathrm{~K}$ \\
$c_{4}$ & $-163.28 \mathrm{~K}$ \\
$c_{5}$ & $-252.73 \mathrm{~K}$ \\
\hline \hline
\end{tabular}

$$
\begin{aligned}
U_{\mathrm{nb}} & =\frac{1}{2} \sum_{i, i^{\prime}=1}^{N_{m}}, \sum_{j, j^{\prime}=1}^{n_{C}} u_{L J}\left(\left|\mathbf{r}_{i j}-\mathbf{r}_{i^{\prime} j^{\prime}}\right|\right)+\sum_{k=1}^{N} u_{g r}\left(\mathbf{r}_{k}\right), \\
U_{\mathrm{b}} & =\sum_{i=1}^{N_{m}}\left[\sum_{j=1}^{n_{C^{-}}} u_{\text {bend }}+\sum_{j=1}^{n_{C^{-}}-3} u_{\text {tors }}+\sum_{j=1}^{n_{C}-4} u_{L J}\right] .
\end{aligned}
$$

\section{RESULTS}

We performed MD simulations as described above for many temperatures in the 100-200 K range. Figure 1 shows a snapshot of typical configurations of the simulated hexane/ graphite system for three characteristic temperatures where the herringbone (HB) commensurate solid, the "nematic," and the fluid phases are observed. It should be mentioned that the promotion of molecules to higher layers was not very significant at any of the temperatures studied. From this figure it is evident that in the low-temperature phase, molecules are arranged in a HB structure where the molecules' azimuthal angles $\phi_{i} \in[0, \pi]$ (Ref. 19) take predominantly values near $\left\{30^{\circ}, 150^{\circ}\right\}$. $\left(\phi_{i}\right.$ is defined as the angle that the axis of the smallest moment of inertia for molecule $i$ makes with the $x$ axis.) Moreover, the molecules are in registry with the substrate. Clearly this HB phase is solid. In the hightemperature regime, it is also quite clear that the molecules are randomly positioned and oriented (and in particular not in registry with the substrate), which is strongly suggestive of a liquid phase. The situation of the mesophase, however, is not as evident. It is clear that it has a definite orientational order, quite different from the HB. [Most azimuthal angles are centered around $0^{\circ}$, the molecules appear not to be commensurate with the substrate, and it is not clear whether the system is in a liquid crystalline state (i.e., a nematic), or in a solid phase (i.e., a rectangular-centered solid).] The exact nature of the different phases (and in particular of the mesophase) is addressed more quantitatively below, by means of the examination of various order parameters, energy analysis, spreading pressure, distributions, and correlation functions.

\section{A. Order parameters}

Figure 2 shows the temperature dependence of several molecular order parameters which are useful in characteriz- 

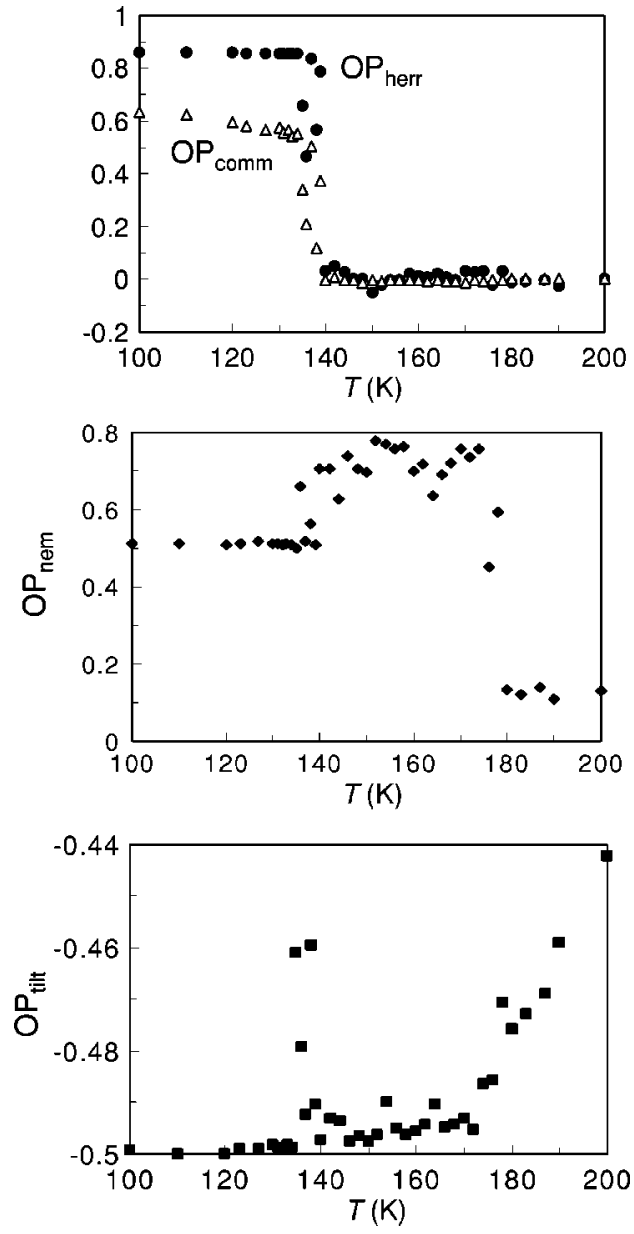

FIG. 2. Structural order information. The following order parameters $(\mathrm{OP})$ are presented as functions of temperature: Herringbone $O P_{\text {herr }}$ (solid circles), center-of-mass $O P_{\text {comm }}$ (open triangles), nematic $O P_{\text {nem }}$ (solid diamonds), and tilt $O P_{\text {tilt }}$ (solid squares).

ing the system's behavior through and across phases. The herringbone order parameter is used to quantify the in-plane orientational ordering of the molecular axes and is defined as

$$
O P_{\text {herr }} \equiv \frac{1}{N_{m}}\left\langle\sum_{i=1}^{N_{m}}(-1)^{j} \sin \left(2 \phi_{i}\right)\right\rangle,
$$

where the sum is peformed over all molecules, the integer $j$ is assigned so as to take the two sublattices into account, and the averages correspond to sampling a large number of uncorrelated configurations in each MD simulation. For a "perfect herringbone" with $\phi_{i} \in\left\{45^{\circ}, 135^{\circ}\right\}$ and $O P_{\text {herr }}=1$, obviously $O P_{\text {herr }}$ vanishes if the two sublattices cannot be distinguished (e.g., if all angles are clustered around one angle or if all angles are sampled equally) either statically (over the lattice) or dynamically (over time). For the HB phase, where the $\phi_{i}$ 's are clustered around $30^{\circ}$ and $150^{\circ}$ (for further evidence see the $130 \mathrm{~K}$ curve in Fig. 7), we find $O P_{\text {herr }} \simeq \sqrt{3} / 2 \simeq 0.866$, as observed for temperatures smaller than $T_{1}=138 \pm 2 \mathrm{~K}$. Not surprisingly $O P_{\text {herr }} \simeq 0$ for the hightemperature phase as essentially all azimuthal angles are equally sampled (see the $200 \mathrm{~K}$ curve in Fig. 7). We also find
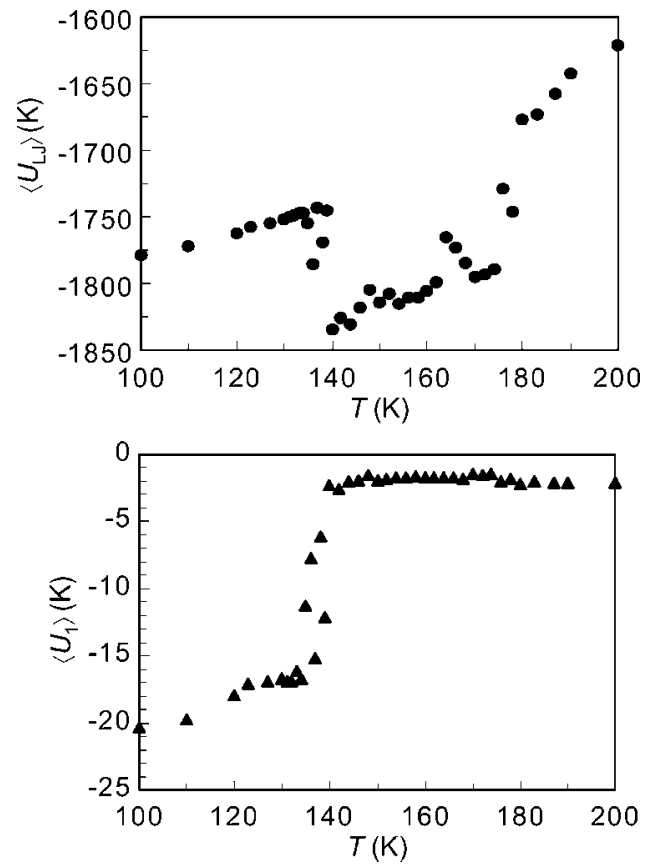

FIG. 3. Thermodynamic information. Ensemble averages per molecule of the Lennard-Jones interaction energy $\left\langle U_{L J}\right\rangle$ and the lateral corrugation in the adsorbate-graphite interaction in Steele's expansion $\left\langle U_{1}\right\rangle$. Note the sharp features of $\left\langle U_{L J}\right\rangle$ in both transitions, and the sharp decrease in magnitude of $\left\langle U_{1}\right\rangle$ at the commensurateincommensurate herringbone-nematic transition.

$O P_{\text {herr }} \simeq 0$ for the mesophase, which is also evident from the $\phi_{i}$ distributions, which show a single significant orientation and thus the lack of two angular distinct sublattices (see the $160 \mathrm{~K}$ curve in Fig. 7).

To investigate whether the adsorbed molecules are in registry with the graphite substrate we also measure the commensurate order parameter $O P_{\text {comm }}$, defined as

$$
O P_{c o m m} \equiv \frac{1}{6 N_{m}}\left\langle\sum_{i=1}^{N_{m}} \sum_{s=1}^{6} e^{-i \mathbf{g}_{s} \cdot \mathbf{r}_{i}}\right\rangle,
$$

where the outer sum runs over all $N_{m}$ molecules at the centerof-mass position $\mathbf{r}_{i}$, and the inner sum runs over all six graphite reciprocal-lattice vectors $\mathbf{g}_{s} \in\{( \pm \pi / 3 a, 0)$, $(\mp \pi / 6 a, \pm \pi / \sqrt{3} a),( \pm \pi / 6 a, \pm \pi / \sqrt{3} a)\} . O P_{\text {comm }}$ takes on a value of unity were all molecular centers to remain statically graphite hexagon centers and vanishes in the limiting case of the molecular centers uniformly sampling positions in the $x y$ plane. As with $O P_{h e r r}$, such a sampling could occur either in a static fashion (an infinite incommensurate solid) or in a dynamic fashion, such as in a nonregistered film. It is evident from Fig. 2, that as the $\mathrm{HB}$ phase disappears at $T_{1}$ $=138 \pm 2 \mathrm{~K}$, the film also loses registry with the substrate. This is also evident in the decrease of the corrugation energy (see Sec. III B and bottom panel of Fig. 3).

Both $O P_{\text {herr }}$ and $O P_{\text {comm }}$ are useful in providing information on the solid-to-nematic transition at $T_{1}=138 \pm 2 \mathrm{~K}$, but they are unable to provide any characterization of the nematic-to-isotropic-liquid transition. The nematic order pa- 
rameter $O P_{\text {nem }}$ provides precisely the needed information and is defined by

$$
O P_{n e m} \equiv \frac{1}{N_{m}}\left\langle\sum_{i=1}^{N_{m}} \cos 2\left(\phi_{i}-\phi_{d i r}\right)\right\rangle,
$$

where $\phi_{d i r}$ is an "average" azimuthal direction defined by maximizing the nematic order parameter: $\partial O P_{n e m} / \partial \phi_{d i r} \equiv 0$,

$$
\phi_{d i r}=\frac{1}{2} \tan ^{-1}\left[\sum_{i=1}^{N_{m}} \sin 2 \phi_{i} / \sum_{i=1}^{N_{m}} \cos 2 \phi_{i}\right] .
$$

Note that due to the $\frac{1}{2}$ prefactor, it is important to use the four-quadrant version of the $\tan ^{-1}$ function to uniquely define $\phi_{d i r}$. (The two-quadrant version leads to the possibility of $\pi / 2$ rotations, which are relevant for the final value of $O P_{n e m}$ in some cases.)

The center panel of Fig. 2 shows the temperature dependence of the nematic order parameter. This order parameter clearly indicates that, in addition to the HB-to-nematic phase transition at $T_{1}=138 \pm 2 \mathrm{~K}$, a second orientational phase transition occurs at $T_{2}=176 \pm 3 \mathrm{~K}$. As shown earlier for the $O P_{\text {herr }}, O P_{\text {nem }}$ also is clearly correlated to the azimuthal angle distributions (Fig. 7). For a perfect herringbone solid $\left(O P_{h e r r}=1\right), \phi_{d i r}$ is completely undefined and $O P_{\text {nem }}$ vanishes, but for the solid, commensurate herringbone phase of hexane on graphite $\left(O P_{h e r r}=\sqrt{3} / 2\right), \phi_{d i r}=0$, and the limiting value of $O P_{\text {nem }}$ is 0.5 . In a perfect nematic phase, all molecules share the same orientation and $O P_{n e m}=1$. Our results of $\max \left[O P_{n e m}\right] \simeq 0.8$ show the presence of significant fluctuations in the molecular orientations and the presence of differently oriented domains. Both features are also clearly qualitatively visible in the $160 \mathrm{~K}$ data presented in the configuration snapshot (Fig. 1) and azimuthal distribution (Fig. $7)$. In the liquid phase we would expect $O P_{\text {nem }}$ to vanish. The small finite value in the high-temperature phase is an indication of a small residual order likely due to the small system size. Note that contrary to the previous order parameters, $O P_{n e m}$ does not vanish due to dynamic fluctuations leading to an overall rotation of the average director over time. We would like to remark that our results for the nematic order parameter (center panel of Fig. 2) differ from those found by Peters and Tildesley $(\mathrm{PT}):^{8}$ (i) they find $O P_{n e m} \simeq-0.5$ for the $\mathrm{HB}$ phase; (ii) they find a relatively small $\max \left[\mathrm{OP}_{\text {nem }}\right]$ $\simeq 0.0-0.4$; (iii) they find the HB-to-nematic transition at a higher temperature (approximately $150 \mathrm{~K}$ ); and (iv) they find a relatively undefined nematic-to-liquid phase transition at approximately $175 \mathrm{~K}$. The difference in (i) [and to some extent (ii)] is due to a factor of $\frac{1}{2}$ difference in the definition of $\phi_{\text {dir }}$ in Eq. (12). The higher "importance" of the nematic mesophase in our work [points (ii) -(iv)] we belive is due to the considerably better statistics in our runs. (Whereas PT used $5 \times 10^{4}$ thermalization and $2 \times 10^{5}$ averaging steps, we typically ran our simulations for $3-5 \times 10^{5}$ thermalization and $0.5-1.0 \times 10^{6}$ averaging steps.)

The tilt order parameter is designed to provide a measure of the out-of-plane orientational order of the system-in particular, the amount of molecular tilting — and is defined as

$$
O P_{\text {tilt }} \equiv \frac{1}{N_{m}}\left\langle\sum_{i=1}^{N_{m}} P_{2}\left(\cos \theta_{i}\right)\right\rangle=\frac{1}{2 N_{m}}\left\langle\sum_{i=1}^{N_{m}}\left(3 \cos ^{2} \theta_{i}-1\right)\right\rangle,
$$

where $P_{2}(x)$ is the Legendre polynomial and $\theta_{i}$ is the angle that the smallest moment of the inertia axis of molecule $i$ makes with the normal to the substrate. Clearly $O P_{\text {tilt }}$ is closely associated with the tilt-angle distribution $P(\theta)$ (see Fig. 8). When all molecules are parallel to the surface $O P_{\text {tilt }}=-0.5$, and $O P_{\text {tilt }}=1$ when all molecules are perpendicular to the surface. The bottom panel of Fig. 2 shows the temperature dependence of $O P_{\text {tilt }}$. At low temperatures the molecules clearly are (as expected) roughly parallel to the graphite substrate to maximize their adsorption energy. At the HB-to-nematic transition, $O P_{\text {tilt }}$ has a significant peak, indicating a tendency of the molecules to librate out of the surface plane, but this tendency is reduced again as the nematic phase is better "established." For increasing temperatures $O P_{\text {tilt }}$ barely increases until the nematic-to-liquid phase transition, where it starts a significantly faster growth. Tilting of the molecules can obvioulsy be a significant factor in the phase transitions, as the "footprint" of the molecules is reduced. To put things in perspective, however, $O P_{\text {tilt }}(200 \mathrm{~K})$ $\simeq-0.44$ roughly corresponds to an average libration of only $11^{\circ}$ out of the plane. Nevertheless, for a full monolayer coverage the resulting "footprint reduction" can be significant. (For more comments on the stifling of tilting by the substrate potential see Sec. III F.)

\section{B. Energy and spreading pressure}

In addition to the structural indicators shown before (the various order parameters), thermodynamic quantities are helpful in tracking phase transitions. Figure 3 shows the temperature dependence of the average Lennard-Jones interaction energy $\left\langle U_{L J}\right\rangle$ [Eq. (2)] and of the average corrugation potential energy $\left\langle U_{1}\right\rangle$ [Eqs. (4) and (5)]. The former is useful in delineating the system's structural behavior irrespective of the substrate's potential, whereas the latter is a good indicator of the atomic order of the substrate and therefore complements the molecular information provided by $O P_{\text {comm }}$ (see Sec. III A).

The average interaction energy is very closely related to how close together the hexane molecules are [in fact, $\left\langle U_{L J}\right\rangle$ $\propto \int u_{L J}(r) g(r) r d r$, where $g(r)$ is the atomic pair correlation function (see Sec. III E)]. The large increase in the magnitude at $T_{1}=138 \pm 2 \mathrm{~K}$ is a clear indication that molecules in the incommensurate, nematic phase have managed to become closer to the minimum of the Lennard-Jones interaction. At the melting transition $\left(T_{2}=176 \pm 3 \mathrm{~K}\right)$ molecular distances again become less optimal and this average energy rises. Both of these features are evident in the plots of $g_{\text {com }}(r)$ (see Fig. 11). The average corrugation potential energy $\left\langle U_{1}\right\rangle$ dramatically decreases in magnitude at $T_{1}$, which is a clear indication of the lack of registration of the adsorbate film and the substrate.

Other energy ensemble averages have interesting features, but considerably less pronounced than those for $\left\langle U_{L J}\right\rangle$ and 


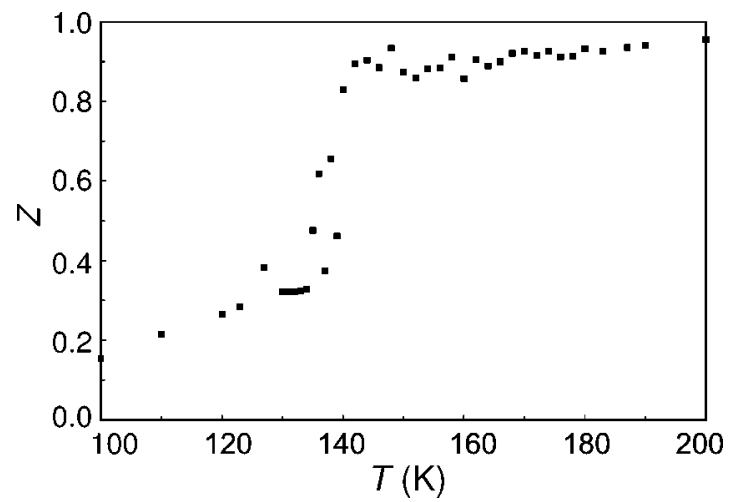

FIG. 4. Relative spreading pressure $Z$ of hexane adsorbed on graphite [see Eq. (14)]. Note that $Z \sim 0$ for $T \leqq T_{1}$, as expected for a solid (small spreading pressure), and that $Z$ approaches the ideal gas value $(Z=1)$ for $T \gtrsim T_{1}$. The value of $Z$ for the intermediate temperature region $\left(T_{1}<T<T_{2}\right)$ indicates that the nematic phase is a fluid phase.

$\left\langle U_{1}\right\rangle$. (Typically they show changes in their derivatives with respect to $T$, but no sudden jumps.) One conclusion that can be drawn from the behavior of these energy averages is that the herringbone commensurate to nematic incommensurate transition is driven by a competition between the intermolecular forces with the substrate corrugation (see Sec. III F for more discussion on this).

The specific heat at constant area $c_{A}$ (not shown) exhibits some scatter, but does present two significant sharp peaks at approximately $138 \mathrm{~K}$ and $176 \mathrm{~K}$, and there is some indication that there may be a latent heat associated with both transitions. However, the energy distributions (not shown) near the transitions do not show any striking bimodality that would seem to suggest that the transition is not first order. As it is usual with finite-size simulations, the determination of the order of the transitions remains elusive.

Another quantity of interest is the spreading pressure (surface "tension") $\Phi$. The virial expansion, ${ }^{20}$

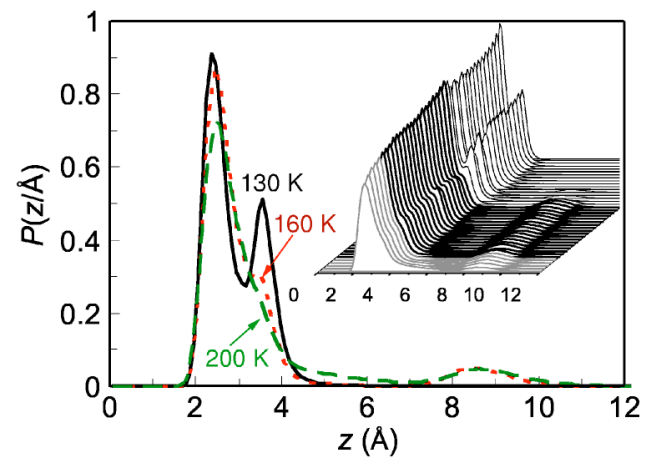

FIG. 5. (Color online) Atomic-height distributions $P(z)$ for the herringbone solid at $T=130 \mathrm{~K}$ (solid black line); nematic at $T$ $=160 \mathrm{~K}$ (red dotted line); and liquid at $T=200 \mathrm{~K}$ (green dashed line). The inset shows all results obtained from $T=100$ to $200 \mathrm{~K}$, back to front, respectively. The thin black lines represent the herringbone $(T \lesssim 138 \mathrm{~K})$, the thick black lines the nematic $(138 \mathrm{~K}$ $\lesssim T \lesssim 176 \mathrm{~K})$, and the gray lines the liquid phases $(T \gtrsim 176 \mathrm{~K})$, respectively.

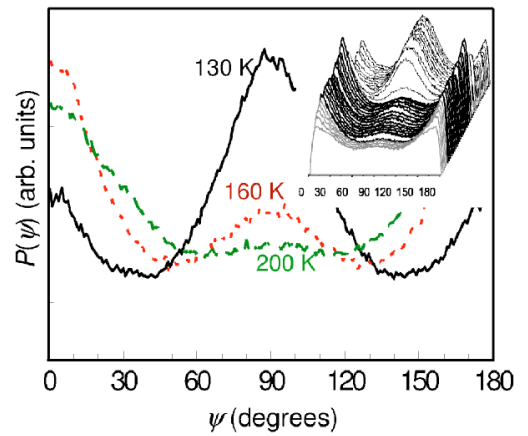

FIG. 6. (Color online) Molecular-roll-angle distributions $P(\psi)$. Please refer to Fig. 5 for format information. Note how sharp the change in $P(\psi)$ is at the HB-to-nematic transition.

$$
\begin{gathered}
\Phi \equiv \frac{N k_{B} T}{A} Z \\
Z \equiv 1+\frac{1}{2 N k_{B} T}\left\langle\sum_{i \neq j}^{N} \mathbf{r}_{i j} \cdot \frac{\partial U}{\partial \mathbf{r}_{i j}}\right\rangle \simeq 1+\int d^{2} r\left[g_{\text {com }}(r)-1\right]
\end{gathered}
$$

relates it to the interactions between molecules or equivalently to the center-of-mass pair correlation function $g_{\text {com }}(r)$ (see Sec. III E). Here N/A is the areal density of the molecules. The "relative spreading pressure" $Z$ is thus a good indicator of how close the equation of state is to that of an ideal gas $(Z \sim 1)$ or that of a solid $(Z \sim 0)$. Figure 4 shows $Z$ for the hexane system. It is evident from our results that below $T_{1}$ the system has a very small spreading pressure as expected for a solid phase, whereas both the nematic $\left(T_{1}\right.$ $\left.<T<T_{2}\right)$ and isotropic phases $\left(T>T_{2}\right)$ are very close to an ideal gas. These results are indicative of the liquid nature of the intermediate nematic phase.

\section{Distributions}

In conjunction to the order parameters mentioned above, various distributions can also help illustrate the properties of the system, especially near the phase transitions, and in par-

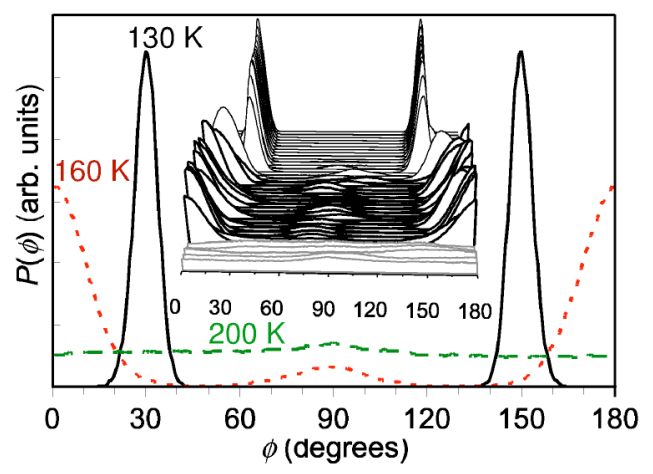

FIG. 7. (Color online) Molecular azimuthal-angle distributions $P(\phi)$. Please refer to Fig. 5 for format information. Note how rapidly the position and shape of the peaks change at the HB-tonematic transition and how fast the peaks disappear in the nematic-to-fluid transition. 


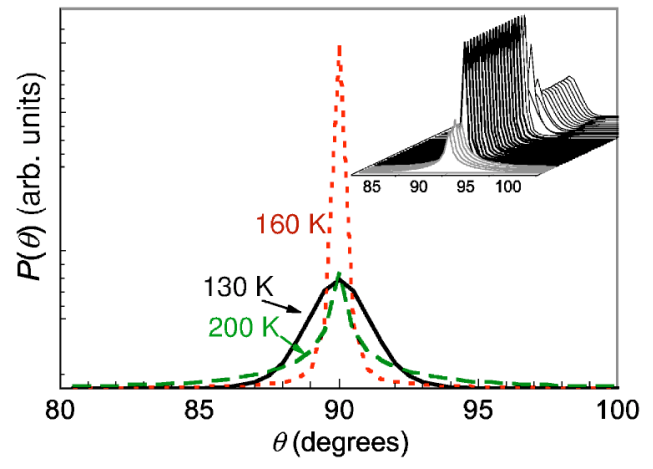

FIG. 8. (Color online) Molecular tilt-angle distributions $P(\theta)$. Please refer to Fig. 5 for format information.

ticular regarding the behavior of various molecular degrees of freedom, i.e., positional, orientational, and internal. In Figs. 5, 6, 7, and 8 we present the atomic height $P(z)$, internal roll-angle $P(\psi)$, azimuthal angle $P(\phi)$, and tilt angle $P(\theta)$ distributions for various temperatures. For $P(z)$ we considered $0 \leqslant z \leqslant 12 \AA$ sampling every $\Delta z=0.0056 \AA$. For $P(\phi)$ and $P(\psi)$ and $P(\theta)$ we considered angles the $\left[0^{\circ}, 180^{\circ}\right]$ domain $\left\{\left[0^{\circ}, 90^{\circ}\right]\right.$ for $\left.P(\theta)\right\}$, sampling every $1^{\circ}$. The azimuthal angle for a molecule $\phi_{i}$ is defined to be the angle formed between the direction of the smallest moment of inertia projected onto the $x y$ plane and the $x$ axis. The tilt angle $\theta$ is defined to be the angle formed between the direction of the smallest moment of inertia and the $z$ axis. $\left(\theta=90^{\circ}\right.$ corresponds to a molecule laying flat on the substrate.) The internal roll angle $\psi$ is defined for a particular bond involving any three consecutive atoms as

$$
\psi=\cos ^{-1}\left\{\frac{\left[\left(\mathbf{r}_{j+1}-\mathbf{r}_{j}\right) \times\left(\mathbf{r}_{j-1}-\mathbf{r}_{j}\right)\right] \cdot \hat{z}}{\left|\left(\mathbf{r}_{j+1}-\mathbf{r}_{j}\right) \times\left(\mathbf{r}_{j-1}-\mathbf{r}_{j}\right)\right|}\right\} .
$$

The roll angle has a value of 0 when the plane containing three consecutive atoms in a molecule is flat (parallel to the graphite basal plane), and it equals $90^{\circ}$ when the plane is perpendicular to the graphite. ${ }^{21}$

The atomic height distribution (Fig. 5) shows that essentially no molecules have been promoted to the second layer in the low temperature phase, and only a very small fraction in the nematic or liquid states. The double peak stucture at low temperatures can be interpreted easily upon consideration of the molecular roll-angle distributions (Fig. 6): as many molecules are rolled "on their side," raising some atoms of the hexane chain above the main peak. At higher temperatures molecules are flatter against the surface and the double-peak in $P(z)$ disappears. The azimuthal angle distribution (Fig. 7) shows three distinct temperature regions corresponding to the HB phase for $T \lesssim 138 \mathrm{~K}[P(\phi)$ peaked at $30^{\circ}$ and $\left.130^{\circ}\right]$, a nematic for $(138 \mathrm{~K} \lesssim T \lesssim 176 \mathrm{~K})[P(\phi)$ peaked at $0^{\circ}$, but note the presence of a small peak near $90^{\circ}$ which corresponds to the formation of domains], and a nearly isotropic fluid for $T \gtrsim 176 \mathrm{~K}$. A discussion of the tilt angle distribution (Fig. 8) is presented later (Sec. IV D), as it is linked to the mechanisms for the phase transitions. See also the discussion on orientational order parameters in Sec. III A.

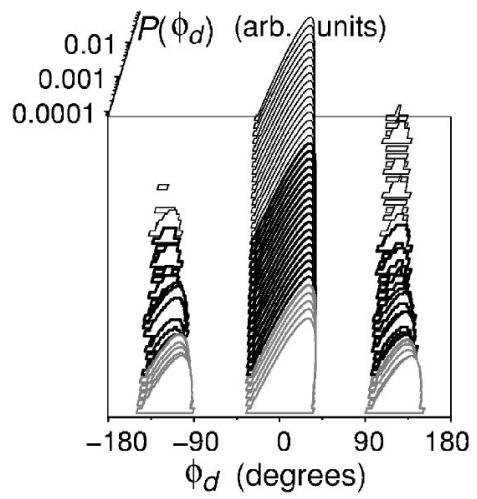

FIG. 9. Dihedral-angle distributions $P\left(\phi_{d}\right)$ for $100 \leqslant T \leqslant 200$ (back to front). Please refer to Fig. 5 for format information.

\section{Internal degrees of freedom}

It is important to monitor the degree to which the phase transitions exhibited by the system are correlated to the internal degrees of molecular freedom. The bond-angle distribution (not shown) is a simple Gaussian corresponding exactly to the Maxwell-Boltzmann distribution for the potential $u_{\text {bend }}$ [Eq. (6)]. This is, in fact, indicative that the bond angles are unaffected by interactions with other molecules or substrates and irrelevant to the properties of the phases and the phase transitions studied. Figures 9 and 10 show the distributions of the dihedral angles $P\left(\phi_{d}\right)$ as well as end-to-end distances $P(d)\left(\mathrm{CH}_{3}-\mathrm{CH}_{3}\right.$ separations in the same molecule), respectively.

The information on both figures is significantly correlated, as the lower-length molecules correspond to the presence of one or more gauche defects. [The width of the main lobe in $P(d)$ corresponds to the width of the bond-angle distribution.] We find that the number of gauche defects is significantly smaller than that for hexane molecules in vacuo [equipartition based on $u_{\text {tors }}$, Eq. (7)], mostly due to the

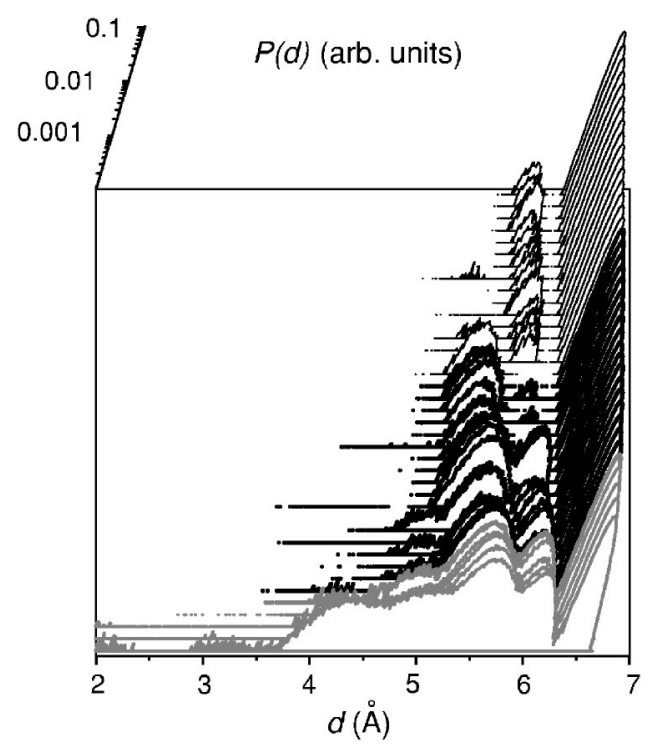

FIG. 10. End-to-end distance distributions $P(d)$ for $100 \leqslant T$ $\leqslant 200$ (back to front). Please refer to Fig. 5 for format information. 
"flattening effect" of the substrate potential, but also due to the interactions with the neighbors, to a lesser degree. The number of gauche defects is small until close to $T_{2}$ and does not present a very significant temperature dependence. The end-to-end probability distribution shows a slightly more pronounced change at $T_{1}$. Given that the fraction of molecules involved (those with gauche defects) is so small, we find it unlikely that these internal degrees of freedom significantly affect the phase transition at $T_{1}$. For the phase transition at $T_{2}$ we find that gauche defects do contribute to the footprint reduction as first suggested by Hansen et al.; 4,5 however, that contribution does not appear to be as important as that from tilting (see Sec. III F).

\section{E. Correlations}

We have also obtained various correlation functions that permit a more complete elucidation of the characteristics of the three phases observed for hexane on graphite to calculate properties that can be directly probed experimentally [i.e., the static structure factor $S(q)$, see below].

We start by defining the center-of-mass molecular paircorrelation function $g_{\text {com }}(r)$, which corresponds to the joint probability of finding molecules with their centers of mass at a distance $r$ away from each other. We also define the orientational pair correlation function $g_{\phi}(r)$, which is calculated by averaging $\cos 2\left(\phi_{j}-\phi_{i}\right)\left(\phi_{i}\right.$ is the azimuthal angle of the $i$ th molecule) calculated over all molecule pairs $(i, j)$ whose centers of mass have a separation $r$. (In practice correlation functions are calculated on a discrete sampling of the distance between the centers of mass; in our case we used $\Delta r$ $=0.083 \AA$, but also note that we used two-dimensional normalization.)

Figure 11 presents $g_{\text {com }}(r)$ and $g_{\phi}(r)$ for various relevant temperatures. For $130 \mathrm{~K}$, both curves confirm that the HBO phase is solid $\left[g_{\text {com }}(r)\right.$ has a sharp peak structure and vanishes between peaks], and the sharp alternating signs of $g_{\phi}(r)$ are indicative of the HBO phase too. At $160 \mathrm{~K}$, however, the correlation functions reveal that the mesophase is, indeed, liquid, but with a very strong orientational order corresponding to a nematic phase. The evidence is quite strong towards labeling the mesophase as a nematic liquid crystal. At $200 \mathrm{~K}$, $g_{\text {com }}(r)$ is rather smooth and $g_{\phi}(r)$ rapidly decays to 0 in a clear indication of a regular liquid behavior. This behavior is consistent with the conclusions obtained from the analysis of the spreading pressure in Sec. III B (see Fig. 4).

A second important correlation function corresponds to the atom-atom pair correlation function $g(r)$, which is defined as the joint probability of finding one atom at a distance $r$ from another (again, distances were discretized with $\Delta r$ $=0.083 \AA$, and we used two-dimensional normalization). Figure 12 shows the atomic pair-correlation function for three characteristic temperatures corresponding to a $\mathrm{HB}$ $(130 \mathrm{~K})$, a nematic $(160 \mathrm{~K})$, and a liquid $(200 \mathrm{~K})$. In all cases, we observe very sharp peaks corresponding to intramolecular pairs; $r=1.54,2.58,3.96,5.16$, and $6.50 \AA$, which correspond exactly to the atomic distances in a hexane molecule in its lowest configurational state. Broader (and weaker) peaks (visible only in the lower panel) at small dis-
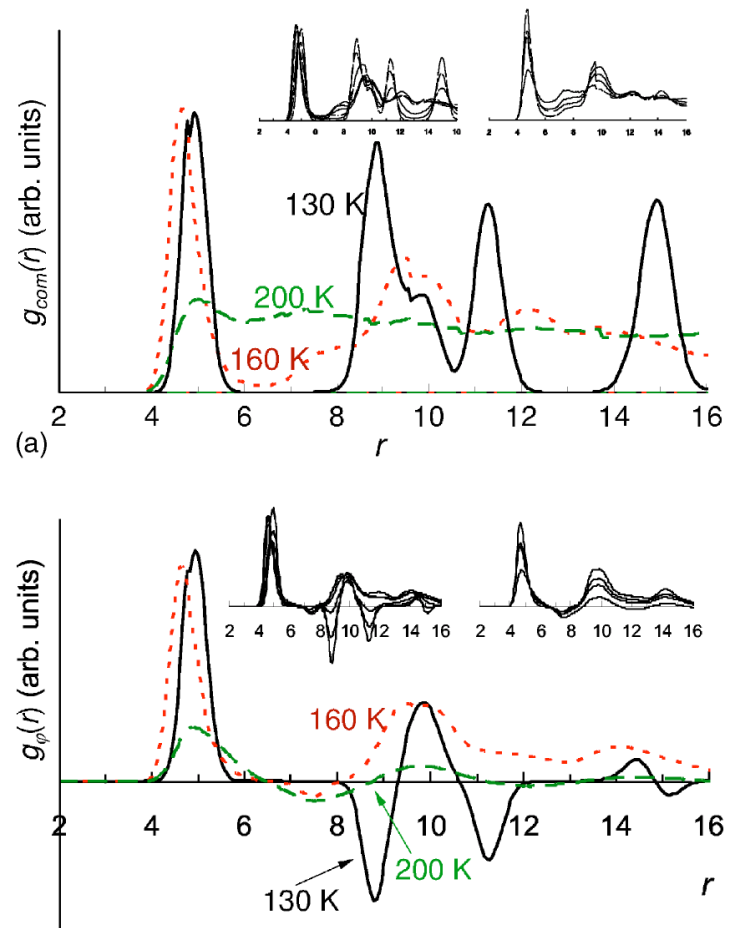

(b)

FIG. 11. (Color online) Center-of-mass molecular paircorrelation function $g_{\text {com }}(r)$ (top panel) and molecular orientational pair-correlation function $g_{\phi}(r)$ for the herringbone solid at $T$ $=130 \mathrm{~K}$ (solid black line); nematic at $T=160 \mathrm{~K}$ (red dotted line); and liquid at $T=200 \mathrm{~K}$ (green dashed line). The left insets show the transitional region between herringbone and nematic $(130 \mathrm{~K}<T$ $<150 \mathrm{~K}$ ), and the right insets show the transitional region between nematic and liquid $(170 \mathrm{~K}<T<180 \mathrm{~K})$.

tances correspond to intramolecular pairs affected by a gauche defect: $r=3.11,4.55$, and $6.05 \AA$. The broad and relatively smooth behavior at larger distances corresponds to pairs belonging to different molecules.

From the atomic pair-correlation function, one can readily obtain the static structure factor $S(q)$, which is very important because it contains spectral weight information in reciprocal space and is therefore directly connected to experimental diffraction data. ${ }^{2-6}$ Since most experiments are performed with powder samples, we chose to calculate a threedimensionally averaged static structure factor,

$$
S(q)=1+\int_{0}^{\infty} \frac{\sin q r}{q r}[g(r)-1] r d r .
$$

Figure 13 presents the static structure factor calculated from the atomic pair-correlation functions in Fig. 12. We note a general broadening of $S(q)$ with increased temperature and only a rather minor shift towards smaller momenta of the main peak. The results are in qualitative agreement with the experimental evidence. ${ }^{2-6}$

\section{F. Variations}

In order to better understand the nature of the phase transitions (in particular the less well known HB to nematic) we 

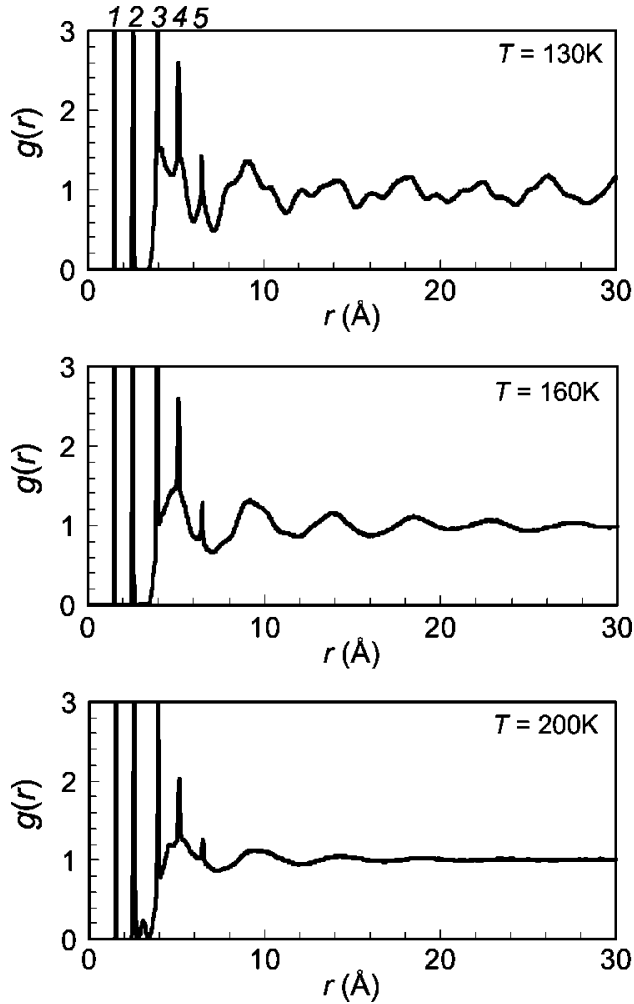

FIG. 12. Atomic pair-correlation function $g(r)$ for the Herringbone solid (top panel, $T=130 \mathrm{~K}$ ), nematic (middle panel, $T$ $=160 \mathrm{~K}$ ), and liquid (bottom panel, $T=200 \mathrm{~K}$ ). The sharp peaks numbered 1-5 correspond to intramolecular pairs at distances corresponding to a hexane molecule in its lowest configurational state: $r=1.54,2.58,3.96,5.16$, and $6.50 \AA$ A. Broader (and weaker) peaks (visible only in the lower panel) at small distances correspond to intramolecular pairs affected by a gauche defect: $r=3.11,4.55$, and $6.05 \AA$. The broad and relatively smooth behavior at larger distances corresponds to pairs belonging to different molecules.

ran MD simulations where some of the interaction potentials were varied. We considered three interesting possibilities: (i) variations of the Lennard-Jones interaction parameters $\epsilon_{\mathrm{CH}_{2}}$ and $\epsilon_{\mathrm{CH}_{3}}$ corresponding to the pseudoatoms of the hexane molecules [Eq. (2)], from $25 \%$ to $200 \%$ of their "standard" values; (ii) variations of the corrugation potential strength in Steele's expansion [Eqs. (4) and (5)], from $25 \%$ to $1000 \%$ of the commonly accepted strength; and (iii) variations of the dihedral potential strength $u_{\text {tors }}\left(\phi_{d}\right)[\mathrm{Eq} .(7)]$ from its usual strength (Table II) to 10 times higher, thus effectively eliminating gauche defects.

We find that for the first type of variation [item (i)], both $T_{1}$ (the HB-to-nematic transition) and $T_{2}$ (the nematic-toisotropic-liquid transition) scale roughly like $\epsilon_{\mathrm{CH}_{\mathrm{n}}}^{1 / 2}$ with a correlation $R^{2} \simeq 0.999$. As $T_{2}-T_{1}$ increases for larger $\epsilon_{\mathrm{CH}_{\mathrm{n}}}$ the nematic has a better chance of "expressing itself," and the maximum value of $O P_{n e m}$ increases too. This simple scaling indicates that the substrate-adatom interaction [mostly through the $E_{0 i}\left(z_{i}\right)$ term-see Eq. (4)—which holds the molecules stronger to the surfaces stifling tilting] is dominant in setting the temperature scales [since the Lorentz-Bertholt rules, Eq. (3), makes this interaction scale like $\left.\epsilon_{\mathrm{CH}_{\mathrm{n}}}^{1 / 2}\right]$. The

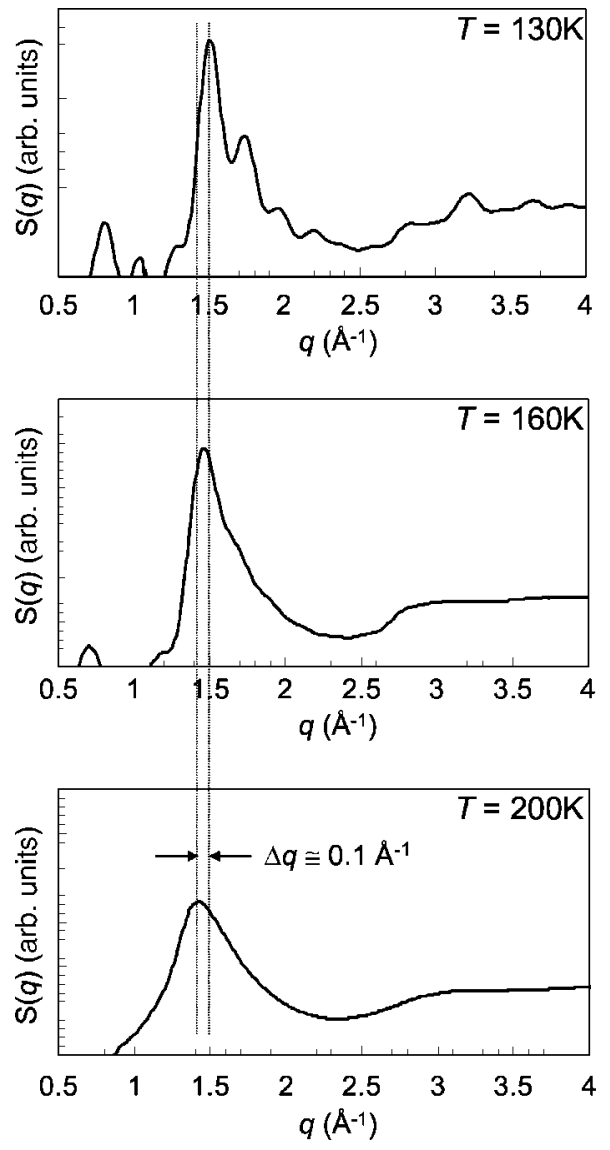

FIG. 13. Static-structure factor $S(q)$ for the Herringbone solid (top panel, $T=130 \mathrm{~K}$ ), nematic (middle panel, $T=160 \mathrm{~K}$ ), and liquid (bottom panel, $T=200 \mathrm{~K}$ ).

reason the holding potential influences the phase transitions directly can be attributed to its stifling effect on tilting and the formation of gauche defects.

In the second type of variation [item (ii)], we find that $T_{1}$ increases with the corrugation energy, whereas $T_{2}$ is almost insensitive to it. As $T_{1}$ increases, the nematic phase becomes "weaker," eventually disappearing for a corrugation approximately 5 times the standard value, and we see a direct transition between the HB and the isotropic fluid. These observations demonstrate that the corrugation is irrelevant for the nematic-to-isotropic melting (as expected, since this transition occurs between two incommensurate phases). Furthermore, the increase of $T_{1}$ is an indication that, in addition to the effect of holding potential described above, a competition between the corrugation potential (which favors a commensurate phase) and the interactions between molecules (which favor some other characteristic intermolecular spacing) is important.

The third type of variation [item (iii)] is useful to characterize which degrees of freedom contribute to the footprintreduction mechanism that creates in-plane space that facilitates the phase transitions. Since the transition at $T_{1}$ happens when the number of gauche defects is minuscule, it is evident that they are not important there. For the phase transition at $T_{2}$ we found (Sec. III) a significant increase in tilting 
and gauche defects as the system achieves its hightemperature phase. Our variation of $u_{\text {tors }}\left(\phi_{d}\right)$, which essentially eliminates gauche defects, resulted in an increase of $T_{2}$ by approximately $20 \mathrm{~K}$. This clearly indicates that the gauche defects are important for footprint reduction as first suggested by Hansen and co-workers, ${ }^{4,5}$ however, tilting appears to be more important.

\section{DISCUSSION}

\section{A. Herringbone-commensurate solid-to-nematic liquid-crystal transition at $T_{1}=138 \pm 2 \mathrm{~K}$}

The low-temperature phase ( $T \lesssim 138 \mathrm{~K}$ ) of the hexane on graphite system is a commensurate, herringbone solid, as evidenced by the structure shown in Fig. 1, the values of $O P_{\text {herr }}, O P_{\text {comm }}$, and $O P_{\text {nem }}$ in Fig. 2, and the two distinct peaks in the azimuthal-angle distribution $P(\phi)$ at $30^{\circ}$ and $150^{\circ}$ in Fig. 7. The spreading pressure of this solid system is very small (low temperature region of Fig. 4). Since the monolayer is complete, the system creates in-plane room by having the molecules spend a significant amount of their time rolled on their sides, as seen in the large peak in the roll-angle distributions $P(\psi)$ in Fig. 6, as well as the doublepeaked atomic-height distribution $P(z)$ (see Fig. 5).

As the temperature of the system is increased, the usual signatures of increasing thermal fluctuations appear, up until $T_{1}=138 \pm 2 \mathrm{~K}$, where the system undergoes a phase change. Together, the structural order parameters in Fig. 2 indicate that the system becomes incommensurate at the same time that it loses its herringbone orientational ordering. In fact, this is also confirmed by the sharp decrease in magnitude in the average Steele corrugation energy $\left\langle U_{1}\right\rangle$ (Fig. 3, bottom panel). This indicates that, even for the atomic degrees of freedom, the system is incommensurate. The center-of-mass pair-correlation function $g_{\text {com }}(r)$ (Fig. 11, top panel) indicates that the new phase is fluid, as evidenced by the fact that $g_{\text {com }}(r)$ has relatively smooth features, and that the molecules are slightly closer to one another, which also leads to an increase in the magnitude of the interaction energy $\left\langle U_{L J}\right\rangle$ (Fig. 3, top panel). The liquid nature of this phase is also evidenced by the relatively large spreading pressure (Fig. 4). As the solid-nematic transition proceeds, the tilt angles of the molecules fluctuate, creating a footprint reduction that facilitates the phase transition (yet tilting is once again reduced when the new phase is reached), as seen by the sharp peak in $O P_{\text {tilt }}$ at approximately $138 \mathrm{~K}$ (Fig. 2, bottom panel).

Inspection of the snapshots in Fig. 1, plus more quantitative considerations based on $O P_{n e m}$ and $P(\phi)$ further indicate that the positional order lost in this phase transition occurs simultaneously with a new orientational order where all molecules have a common director. The molecules experience, in fact, a relatively long-range orientational order (see $160 \mathrm{~K}$ curve, Fig. 11, bottom panel). We believe that this phase has all the characteristics of a two-dimensional nematic liquid crystal. The analysis of the probability distributions for the bond and dihedral angles and that for the molecular end-toend distance indicate that the internal degrees of freedom do not cooperate significantly with the HB-to-nematic phase transition. In fact, it seems that they hardly participate at all in the relevant processes in the temperature range studied for a monolayer of hexane adsorbed on graphite. It should be noted that the number of gauche defects is significantly smaller than what would be predicted in vacuo, which is due to a large degree to the substrate interaction and to a lesser degree to the interaction between molecules, which has an aligning effect.

\section{B. Nematic-to-isotropic-liquid phase transition at $T_{2}=176 \pm 3 \mathrm{~K}$}

As the temperature is raised, the nematic phase persists until $T_{2}=176 \pm 3 \mathrm{~K}$, where another orientational phase transition occurs, this time to an isotropic fluid. The phase transition is characterized by a sudden drop in the nematic order parameter $O P_{\text {nem }}$ (Fig. 2, center panel) and the disappearance of any directional preference in the azimuthal-angle distribution $P(\phi)$ (Fig. 7, see also the random orientation in the $200 \mathrm{~K}$ snapshot in Fig. 1). In addition, the orientational paircorrelation function $g_{\phi}(r)$ shows that the angular correlations between molecules decay extremely rapidly with distance in the high-temperature phase (see the $200 \mathrm{~K}$ curve, Fig. 11, bottom panel). The Lennard-Jones interaction increases sharply (which is related to the increase in the typical intermolecular distances), and there is a strong peak at $T_{2}$ in the specific heat $c_{A}$.

In the liquid, the tilt-angle behavior shown in the bottom panel of Fig. 2 reveals that the molecular axes are much freer to fluctuate than in the nematic phase, and this may cause a significant reduction in the molecular footprint. (As discussed in Sec. III F, gauche defects also contribute to this footprint reduction, though tilting appears to be more important.) The dihedral angle and end-to-end distance distributions in Figs. 9 and 10 show that, in the liquid phase, gauche and other dihedral defects are promoted more readily with increasing temperature, but, as with the solid-to-nematic phase transition, no new or abrupt changes in the internal degrees of molecular freedom accompany melting.

\section{Information related to scattering}

The atomic pair-correlation functions in Fig. 12 are a combination of intramolecular and intermolecular pair terms and, hence, are rich in information about how the internal degrees of freedom behave as well as the bulk structure of the system's phase. The five extremely sharp peaks correspond to atom-atom distances within the molecules, and then correspond precisely to the intramolecular atomic distances in the ground state of hexane. A careful inspection of the $g(r)$ curves at high temperatures reveals the emergence of smaller, more diffuse peaks, which again precisely correspond to the intramolecular atomic distances, this time in the presence of one gauche defect. When the curve $g(r)$ starts to elevate off the horizontal axis, the contribution from atom 
pairs between different molecules begins, and the decreasing structural order with increasing temperature is evident. The static structure factor $S(q)$ shown in Fig. 13 helps us to further understand the behavior of $g(r)$. We note a general broadening of $S(q)$ with increased temperature and only a rather minor shift towards the smaller momenta of the main peak. The results are in qualitative agreement with experimental evidence..$^{2-6}$

\section{Mechanisms}

As in previous computer simulations, ${ }^{7-9}$ our work reproduces the general features of the real system as explored by experiment and quite accurately determines the melting temperature $T_{2}{ }^{1-6}$ Our findings of an intermediate nematic liquid crystalline phase between $T_{1}$ and $T_{2}$ are roughly in agreement with previous simulations by Peters and Tildesley. ${ }^{8}$ However, the existence of this intermediate phase has not been confirmed experimentally, which suggests that larger computer simulations would be of interest in determining the presence of highly complicated domain behavior or even dynamic domains that smaller simulations simply cannot capture.

A unique benefit of computer simulations is that they allow a careful inspection of the mechanism for various phase transitions when enough data are present. In the work presented here, we see that in the solid herringbone phase the molecules spend much time rolled, so that their backbones are zigzagged or rough, relative to the graphite substrate (see Fig. 6). The solid-to-nematic transition involves a negligible number of gauche defects (Fig. 9), instead showing pronounced molecular tilt fluctuations (Fig. 2, bottom panel) in concert with the molecules' rolling on their sides. The resulting nematic phase still has an average density of unity, but the molecules are closer to each other (in the direction perpendicular to the molecules), as evidenced by the shift in the first peak of the center-of-mass pair-distribution function $g_{\text {com }}(r)$ (Fig. 11) and the sudden increase in magnitude of the Lennard-Jones interaction $\left\langle U_{L J}\right\rangle$ (Fig. 3, top panel). As a result, the tilt-angle distribution (Fig. 8) takes on a fundamentally different shape, somewhat more sharply peaked than the Gaussian-like distributions seen in the solid. The importance of the molecule-molecule interaction in the mesophase is underscored by the fact that the tilt-angle distributions do not considerably broaden in the nematic.

At a higher temperature the system melts onto an isotropic phase. Although no sudden increase in tilt fluctuation accompanies melting, the tilt-angle distributions begin to show thermal broadening, accounting for the increased slope of $O P_{\text {tilt }}$ in the liquid (Fig. 2, bottom panel). A possible "signature" of the nematic is that the tilt angles are locked and that it is a "tilt-locking" mechanism that preceeds the mesophase. The first simulations completed on the system ${ }^{4,5}$ concluded that, at melting, the proliferation of the dihedral (gauche) defects in the adsorbate cause the projected presence of the molecules (their footprint) to decrease, thus creating more in-plane room for center-of-mass molecular fluctuations and thus allowing melting to ensue. In fact, the freezing of the dihedral degrees of freedom resulted in a considerable increase in the system's melting temperature. Later work by the same authors ${ }^{6}$ and others ${ }^{7-9}$ suggests that, when the molecule-substrate interaction strength is decreased to more realistic values, the gauche defects become unimportant for melting. Our work suggests that the creation of inplane free space is indeed important for all phase transitions in the system, but that the footprint reduction happens exclusively through tilting (and moderate stacking) at the solidnematic transition, where the internal molecular degrees of freedom are unimportant. The footprint reduction for the nematic-isotropic transition is, indeed, caused by both the gauche defects and the tilting, as both the probability of finding significant dihedral angles and tilting start rising significantly near $T_{2}$ (see the bottom panel of Fig. 2 and Fig. 9).

The simulations conducted on the variation of various Lennard-Jones interaction parameters agree with the above assessments, because when only the lateral graphite corrugation well depth is varied [type (ii) as defined in Sec. III F], $T_{1}$ increases slightly and $T_{2}$ remains unchanged. However, when the potential parameters for the pseudoatoms themselves are varied [type (i)], the interactions of the adatoms with the substrate also increase, including a stronger vertical-force component. Thus, tilting is stifled and $T_{1}$ and $T_{2}$ are significantly affected. Since we already know that an increase in lateral forces alone causes relatively small changes in $T_{1}$, such results underscore the importance of tilting prior to the nematic. Our elimination of gauche defects by varying the torsional potential [variation type (iii)] resulted in a moderate increase in the transition temperature $T_{2}$, from which we conclude that both tilting and gauche defects are significant, albeit it seems that the former are more important. In essence, we agree qualitatively with the earlier works' findings that footprint reduction is important in this system, only in our work, the prevalent mechanism is tilting.

\section{E. Final remarks}

Our simulations indicate that the HB-to-nematic transition is due to a competition between the procommensurate interaction between adsorbate molecules and the corrugation substrate potential and the intermolecular interactions which favor intermolecular distances different from those required by the substrate. The latter prevail at approximately $138 \mathrm{~K}$, leading to a nematic liquid crystalline state. If a stronger attraction to the substrate is used in our similations (Sec. III F), the stifling of mechanisms that reduce the molecular footprint makes the phase transition occur at a higher temperature. An increase in the corrugation potential has a similar effect. The nematic-to-isotropic transition is, as expected, rather insensitive to the corrugation potential, but it remains very sensitive to the main part of the substrate-adatom potential.

We have observed somewhat contradictory evidence in trying to identify the order of the phase transitions. Whereas the specific-heat curves seem to indicate that there may be a latent heat in both cases, the lack of bimodality in the energy distributions would indicate otherwise. The inconclusiveness 
is not, however, surprising, given the finite size of the system simulated.

We have also performed MD simulations with approximately $2 \times$ and $4 \times$ the number of molecules discussed throughout this paper. We find that the HB-to-nematic phase transition temperature $T_{1}$ is only weakly dependent on system size (but the relatively poor statistics possible with the larger sizes make it difficult to extrapolate to the thermodynamic limit). The nematic-to-isotropic phase transition temperature $T_{2}$ is insensitive to system size for the sizes considered.

\section{ACKNOWLEDGMENTS}

The authors are indebted to Haskell Taub, Flemming Hansen, Güenther Peters, Peter Pfeifer, Cintia Lapilli, and James MacCollough for enriching discussions. Acknowledgment is made to the Donors of the Petroleum Research Fund, administered by the American Chemical Society, for support of this research. CW acknowledges support from the University of Missouri Research Board and the University of Missouri Research Council. MR and CP acknowledge support by the University of Northern Iowa.
${ }^{1}$ J. Krim, J. Suzanne, H. Shechter, R. Wang, and H. Taub, Surf. Sci. 162, 446 (1985).

${ }^{2}$ J. C. Newton, Ph.D. dissertation, University of MissouriColumbia, 1989.

${ }^{3} \mathrm{H}$. Taub, in NATO Advanced Study Institutes, Series C: Mathematical and Physical Sciences, edited by G. J. Long and F. Grandjean (Kluwer, Dordrecht, 1988), Vol. 228, pp. 467-497.

${ }^{4}$ F. Y. Hansen and H. Taub, Phys. Rev. Lett. 69, 652 (1992).

${ }^{5}$ F. Y. Hansen, J. C. Newton, and H. Taub, J. Chem. Phys. 98, 4128 (1993).

${ }^{6}$ K. W. Herwig, Z. Wu, P. Dai, H. Taub, and F. Y. Hansen, J. Chem. Phys. 107, 5186 (1997).

${ }^{7}$ E. Velasco and G. H. Peters, J. Chem. Phys. 102, 1098 (1995).

${ }^{8}$ G. H. Peters and D. J. Tildesley, Langmuir 12, 1557 (1996).

${ }^{9}$ G. H. Peters, Surf. Sci. 347, 169 (1996).

${ }^{10}$ J. M. Kosterlitz and D. J. Thouless, J. Phys. C 6, 1181 (1973).

${ }^{11}$ J. M. Kosterlitz, J. Phys. C 7, 1096 (1974).

${ }^{12}$ B. I. Halperin and D. R. Nelson, Phys. Rev. Lett. 41, 121 (1978).

${ }^{13}$ D. R. Nelson and B. I. Halperin, Phys. Rev. B 19, 2457 (1979).
${ }^{14}$ K. J. Strandburg, Rev. Mod. Phys. 60, 161 (1988).

${ }^{15}$ M. P. Allen and D. J. Tildesley, Computer Simulation of Liquids (Clarendon Press, New York, 1988).

${ }^{16}$ M. G. Martin and J. I. Siepmann, J. Phys. Chem. 102, 2569 (1998).

${ }^{17}$ W. A. Steele, Surf. Sci. 36, 317 (1973).

${ }^{18}$ P. Padilla and S. Toxværd, J. Chem. Phys. 94, 5650 (1991).

${ }^{19}$ Note that, since hexane is not polar, we are dealing with directors rather than vectors, hence the $[0, \pi]$ range for $\phi_{i}$. Similar arguments also apply to other molecular directions $\left(\theta_{i} \in[0, \pi / 2], \psi\right.$ $\in[0, \pi])$.

${ }^{20}$ See, e.g., M. Plischke and B. Bergersen, Equilibrium Statistical Physics, 2nd. ed. (World Scientific, Singapore, 1994), chaps. 4 and 7.

${ }^{21}$ The internal roll angle is related to the rolling angle in the previous work (Refs. 8 and 9), with the exception that it gives information about the rotation of the plane that each bond angle lies in, as opposed to the overall molecular orientation. 